\title{
Transient Radiative and Conductive Heat Transfer in Ceramic Materials Subjected to Laser Heating ${ }^{1}$
}

\author{
M. Musella ${ }^{2,3}$ and H. R. Tschudi ${ }^{2}$
}

\begin{abstract}
In this work the problem of transient energy transport in a conductive, emitting, and scattering medium is addressed. A new approach for describing the transient response of a participating one-dimensional layer subjected to a finite laser pulse is presented. The model considers heat transport in one dimension by conduction and radiation, where the radiative field is described by the two-flux approximation. The material is assumed to be gray, homogeneous, and isotropic, with physical and optical properties dependent on temperature. Numerical results have been obtained on a layer of low optical thickness using the thermophysical properties of zirconia as a typical material.
\end{abstract}

KEY WORDS: emitting and scattering material; isotropic; participating medium; semitransparent; two-flux model.

\section{INTRODUCTION}

Ceramics have excellent heat and corrosion resistant properties, and they are nowadays extensively employed both as structural and coating materials for high-tech applications, especially at very high temperatures. Some of these materials, such as zirconia, are partially transparent to radiant energy. Internal absorption, emission, and scattering of thermal radiation have therefore strong influence on their internal temperature distributions.

For a proper description of energy transfer in those materials, detailed modeling of the interaction of light with matter and of the combined

\footnotetext{
${ }^{1}$ Paper presented at the Seventh International Workshop on Subsecond Thermophysics, October 6-8, 2004, Orléans, France.

${ }^{2}$ Paul Scherrer Institut, CH-5232 Villigen-PSI, Switzerland.

${ }^{3}$ To whom correspondence should be addressed. E-mail: manuela.musella@psi.ch
} 
effects of radiative and conductive heat transfer is required. During recent years radiative transfer has become the subject of many investigations, and because of the mathematical complexity of the topic, several approximation techniques have been developed to treat the problem [1]. Most of the studies, however, deal with steady-state solutions, and the computational complexity of the time-dependent problems restricted studies of transient behavior to very special cases $[2,3]$. The problem of transient coupled radiative and conductive heat transfer for scattering materials was addressed by only a few authors, and also in this case, only very special problems were solved [4-6].

This work addresses the problem of energy transport by conduction and radiation in a participating medium (absorbing, emitting, and scattering) subjected to an impinging time-dependent flow of radiating energy, and proposes a new approach for approximately solving the two-flux equations [7] describing the radiative field. This approximate treatment rests on the observation that the two-flux equations describing the radiative field are inhomogeneous, linear differential equations. The general solution of these equations is expressed as the sum of a special solution of the inhomogeneous equations and of the general solution of the homogeneous equations, the equations without the emission term. Of those two solutions, the first accounts for the emission term, while the second matches the boundary condition for the radiative field. A special solution of the inhomogeneous equations is found here by a systematic formal expansion, whereas the homogeneous two-flux equations can be solved analytically for constant material parameters.

We developed the procedure for describing the response of an isotropic, gray, polycrystalline ceramic layer subjected to a laser pulse impinging on one side, the other being kept at constant temperature. The model takes into account energy transport in one dimension by conduction and radiation with temperature-dependent material properties. Results are shown for a layer of zirconia, a typical high porosity absorbing and scattering material.

\section{PHYSICAL MODEL AND GOVERNING EQUATIONS}

An infinitely extended plane layer of thickness $L$, consisting of a conductive, gray emitting, absorbing, and scattering medium is considered here. The layer, initially at a temperature $T_{L}$, is uniformly irradiated on the front surface by a laser pulse of finite width, while its rear surface is kept at a constant temperature $T_{L}$. The system is placed in vacuum. The material is assumed to be homogenous and isotropic, with physical and optical properties that may depend on temperature. 
Treating the problem as one dimensional (i.e., temperature and energy fluxes vary only in the direction perpendicular to the surfaces), the transient energy equation describing the system has the form,

$$
d(T) c_{p}(T) \frac{\partial T(x, t)}{\partial t}=-\frac{\partial}{\partial x}\left(J_{Q}+J_{\mathrm{rad}}\right)
$$

For solving Eq. (1) the conductive and radiative flux have to be related to the instantaneous temperature and light distribution inside the material. While the conductive flux $J_{Q}$ can be expressed by Fourier's equation,

$$
J_{Q}=-k(T) \frac{\partial T(x, t)}{\partial x},
$$

such a formula does not exist for describing the radiative flux. The formulation and solution of the exact equations of radiative transfer including scattering is rather complicated, and usually approximation methods are used to simplify the problem. It has been extensively demonstrated [1] that the two-flux approximation provides an accurate simplified formulation for describing the present case, and it will therefore be used here. As shown in Fig. 1, a two-flux model assumes that the radiative field in the medium is described by only two uniform fluxes, $\mathbf{J}_{+}$and $\mathbf{J}_{-}$, the first flowing in the positive $x$-direction and the second in the negative $x$-direction. According to the Kubelka-Munk theory [8], the equations governing these two quantities can be written as

$$
\begin{gathered}
-\frac{\partial J_{-}}{\partial x}=S J_{+}-(A+S) J_{-}+q, \\
\frac{\partial J_{+}}{\partial x}=-(A+S) J_{+}+S J_{-}+q,
\end{gathered}
$$

where $q$ is the source term, which for a gray body is expressed by

$$
q=A \sigma_{B} T(x)^{4} .
$$

Introducing the quantity,

$$
J_{\text {rad }}(x, t)=J_{+}(x, t)-J_{-}(x, t)
$$

and

$$
F(x, t)=J_{+}(x, t)+J_{-}(x, t)
$$


it follows from Eqs. (3) and (4) that

$$
\frac{\partial J_{\mathrm{rad}}(x, t)}{\partial x}=-A\left(F(x, t)-2 \sigma_{B} T^{4}\right) .
$$

and

$$
\frac{\partial F(x, t)}{\partial x}=-(A+2 S) J_{\operatorname{rad}}(x, t) .
$$

For given boundary conditions, the solution of the equations of radiative transfer, Eqs. (5) and (6), can be expressed as the superposition of a special solution of the full, inhomogeneous equation and a solution of the homogeneous equation without the source term, $A \sigma_{\mathrm{B}} T^{4}$, so that $J_{\mathrm{rad}}$ and $F$ can be expressed as

$$
J_{\mathrm{rad}}=J^{\mathrm{si}}+J^{\mathrm{h}}
$$

and

$$
F=F^{\mathrm{si}}+F^{\mathrm{h}} \text {. }
$$

The first terms on the right-hand side of Eq. (7), $J^{\text {si }}$ and $F^{\text {si }}$, take into account the source term while with the second contributions, $J^{\mathrm{h}}$ and $F^{\mathrm{h}}$, are used to match the boundary conditions for the radiative field.

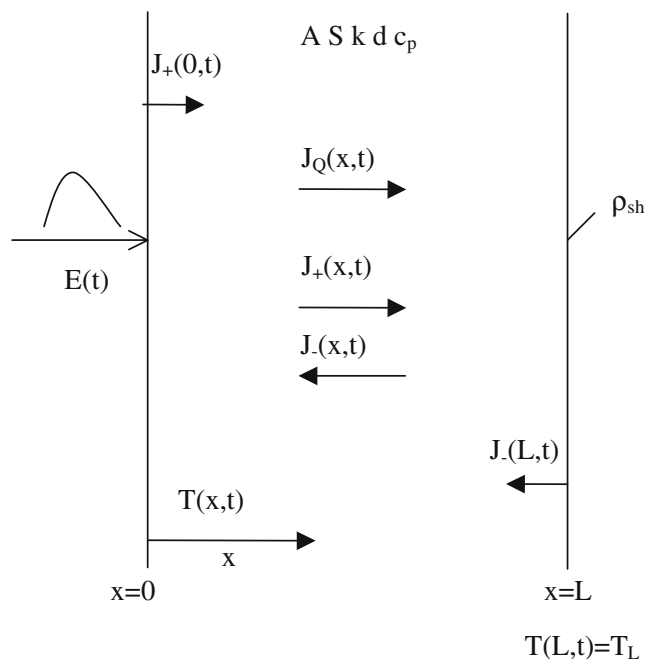

Fig. 1. Geometry, coordinate system, and nomenclature of the studied physical system. 
A special solution of the inhomogeneous equation can be derived from Eqs. (5) and (6) recursively. Starting with the value of $J(x)=0$, one gets for the first recursion from Eq. (5),

$$
F^{\mathrm{si}}=2 \sigma_{\mathrm{B}} T^{4}
$$

which substituted in Eq. (6) gives

$$
J^{\mathrm{si}}=-\frac{8 \sigma_{\mathrm{B}} T^{3}}{A+2 S} \frac{\partial T}{\partial x} .
$$

The recursion does not need to be driven further, and Eqs. (8a) and (8b) are accepted as the special solution of the inhomogeneous two-flux equations. It should also be noted that Eq. (8b) corresponds to the diffusion approximation [6].

The system of Eqs. (5) and (6) without the source term (homogeneous equations) can be written as

$$
\begin{gathered}
\frac{\partial J^{\mathrm{h}}(x, t)}{\partial x}=-A F^{\mathrm{h}}(x, t) \\
\frac{\partial F^{\mathrm{h}}(x, t)}{\partial x}=-(A+2 S) J^{\mathrm{h}}(x, t),
\end{gathered}
$$

which can be solved analytically when $A$ and $S$ do not vary with $x$. Since the solution of the homogeneous equations is important only near the surface, it is reasonable to use this analytical solution with the boundary values of $A$ and $S$ appropriately chosen (the full temperature dependence of these quantities can be still used in the special solution of the inhomogeneous two-flux equations)

The analytical solution of the system of Eq. (9) is thus expressed as

$$
F^{\mathrm{h}}(x)=-\frac{W}{A} \sinh (W x) J^{\mathrm{h}}(0)+\cosh (W x) F^{\mathrm{h}}(0)
$$

and

$$
J^{\mathrm{h}}(x)=\cosh (W x) J^{\mathrm{h}}(0)-\frac{A}{W} \sinh (W x) F^{\mathrm{h}}(0),
$$

where $W=\sqrt{A^{2}+2 A S}$ and $J^{\mathrm{h}}(0)$ and $F^{\mathrm{h}}(0)$ depend on $J_{+}^{\mathrm{h}}(0, t)$ and $J_{-}^{\mathrm{h}}(L, t)$, the radiative fluxes at the boundaries. For determining these two quantities, let's consider the boundary conditions for the present case.

On the front surface the layer is irradiated by a laser pulse of power $E(t)$. The layer conducts heat internally, but because of the vacuum surroundings, there is no mechanism by which heat can be conducted away 
from the boundary. From $J_{Q}(0, t)=0$ it follows that $\partial T / \partial x(0, t)=0$ and thus $J^{\mathrm{si}}(0, t)=0$. Hence, the radiative balance at the front surface is

$$
E(t)=J_{+}(0, t)=\frac{1}{2}\left[F^{h}(0, t)+2 \sigma_{\mathrm{B}} T^{4}(0, t)+J^{h}(0, t)\right] .
$$

The rear surface of the layer is kept at constant temperature $T(L, t)=T_{L}$ by a thermostat having a reflectivity $\rho_{s h}$. The radiative balance at the rear surface can be thus written as

$$
J_{-}(L, t)=\rho_{s h} J_{+}(L, t)+\left(1-\rho_{s h}\right) \sigma_{\mathrm{B}} T_{L}^{4}
$$

that with

$$
\theta=\frac{1-\rho_{s h}}{1+\rho_{s h}}
$$

yields the expression,

$$
\theta F^{\mathrm{h}}(L, t)-J^{\mathrm{h}}(L, t)=J^{\mathrm{si}}(L, t) .
$$

Substituting Eqs. (11) and (13) in Eq. (10) calculated at $x=L$, it follows that

$$
\begin{aligned}
J^{\mathrm{h}}(0) & =\frac{2\left[\theta \cosh (W L)+\frac{A}{W} \sinh (W L)\right]\left(E-\sigma_{B} T_{0}^{4}\right)-J_{L}^{\mathrm{si}}}{(1+\theta) \cosh (W L)+\left[\frac{A}{W}+\theta \frac{W}{A}\right] \sinh (W L)}, \\
F^{\mathrm{h}}(0) & =\frac{2\left[\cosh (W L)+\theta \frac{W}{A} \sinh (W L)\right]\left(E-\sigma_{B} T_{0}^{4}\right)+J_{L}^{s i}}{(1+\theta) \cosh (W L)+\left[\frac{A}{W}+\theta \frac{W}{A}\right] \sinh (W L) .}
\end{aligned}
$$

Equations (1), (2), (5)-(8), (10), and (14) represent the complete system of equations that allows a solution to the problem of transient energy transfer for the physical system mentioned above.

For the numerical evaluation of Eqs. (10) and (14), one has to take care of numerical extinction. For $W L \gg 1$, these equations may not be used for the calculation in the present form and the hyperbolic sines and cosines have to be resolved into the corresponding exponential functions.

It should be noted that the above results have been derived using the Kubelka-Munk approximation, but the same approach can be applied with similar results for any two-flux approximation of the radiative field as, for instance, the Milne-Eddington or the Schuster-Schwarzschild approximations. 


\section{NUMERICAL SOLUTION PROCEDURE}

The combined one-dimensional conductive-radiative problem just described was solved numerically. For convenience Eq. (1) is rewritten as

$$
d(T) c_{p}(T) \frac{\partial T(x, t)}{\partial t}=-\frac{\partial}{\partial x}\left(J_{Q}+J^{\mathrm{si}}+J^{\mathrm{h}}\right)=-\frac{\partial}{\partial x}\left(J_{Q}+J^{\mathrm{si}}\right)-\frac{\partial J^{\mathrm{h}}}{\partial x}
$$

with Eqs. (2), (8b), and (9a), it follows that Eq. (1) has the form,

$$
d(T) c_{p}(T) \frac{\partial T(x, t)}{\partial t}=-\frac{\partial J}{\partial x}+A F^{\mathrm{h}},
$$

where

$$
J=-k_{h r t}(T) \frac{\partial T(x, t)}{\partial x}
$$

and

$$
k_{h r t}(T)=k(T)+\frac{8 \sigma_{B}}{A+2 S} T^{3},
$$

which is the classical transient energy equation, where the first term of Eq. (15) is analogous to Fourier's law, and the term $\mathrm{AF}^{\mathrm{h}}$ represents a source term.

For numerically solving Eq. (15) the investigated layer was divided into $N$ cells (Fig. 2), the cells indexed with $n$, with $0 \# n \# N$. Each cell was assign the temperature calculated at $\left(x_{n}+x_{n-1}\right) / 2$.

The energy flux $J_{n}$ flowing from cell $n$ to cell $n+1$ is given by two expressions,

$$
J_{n}=-\frac{k_{h r t, n}}{\delta_{n} / 2}\left(T_{n}^{*}-T_{n}\right)=-\frac{k_{h r t, n+1}}{\delta_{n+1} / 2}\left(T_{n+1}-T_{n}^{*}\right),
$$

where $T_{n}^{*}$ is the temperature at the boundary $n / n+1$. Eliminating $T_{n}^{*}$ from Eq. (18),

$$
J_{n}=-2 \frac{k_{h r t, n} k_{h r t, n+1}}{\delta_{n} k_{h r t, n+1}+\delta_{n+1} k_{h r t, n}}\left(T_{n+1}-T_{n}\right) .
$$

The discrete analogous part of Eq. (15) is thus

$$
\frac{\Delta T_{n}}{\Delta t}=\frac{1}{\left(d c_{p}\right)_{n}} \frac{1}{\delta_{n}}\left(J_{n-1}-J_{n}\right)+\frac{1}{\left(d c_{p}\right)_{n}} A_{n} F_{n}^{\mathrm{h}},
$$

where $F_{n}^{\mathrm{h}}$ is determined from Eq. (10a) at $x=x_{n}$. 
With the definitions,

$$
D_{n}=\frac{1}{\left(d c_{p}\right)_{n} \delta_{n}} \text { and } \eta_{n}=\frac{2 k_{h r t, n} k_{h r t, n+1}}{\delta_{n+1} k_{h r t, n}+\delta_{n} k_{h r t, n+1}} .
$$

Eq. (20) reads as

$$
\frac{\Delta T_{n}}{\Delta t}=D_{n} \eta_{n-1} T_{n-1}-D_{n}\left(\eta_{n}+\eta_{n-1}\right) T_{n}+D_{n} \eta_{n} T_{n+1}+q_{n}
$$

with the source term,

$$
q_{n}=\frac{A_{n}}{\left(d c_{p}\right)_{n}} F_{n}^{\mathrm{h}} .
$$

Equation (21a) is valid for the interior points $2 \# n \# N-2$.

As explained earlier at the boundary $n=0$, the temperature gradient and thus $J_{0}$ are zero, yielding

$$
\frac{\Delta T_{1}}{\Delta t}=-D_{1} \eta_{1} T_{1}+D_{1} \eta_{1} T_{2}+q_{1}
$$
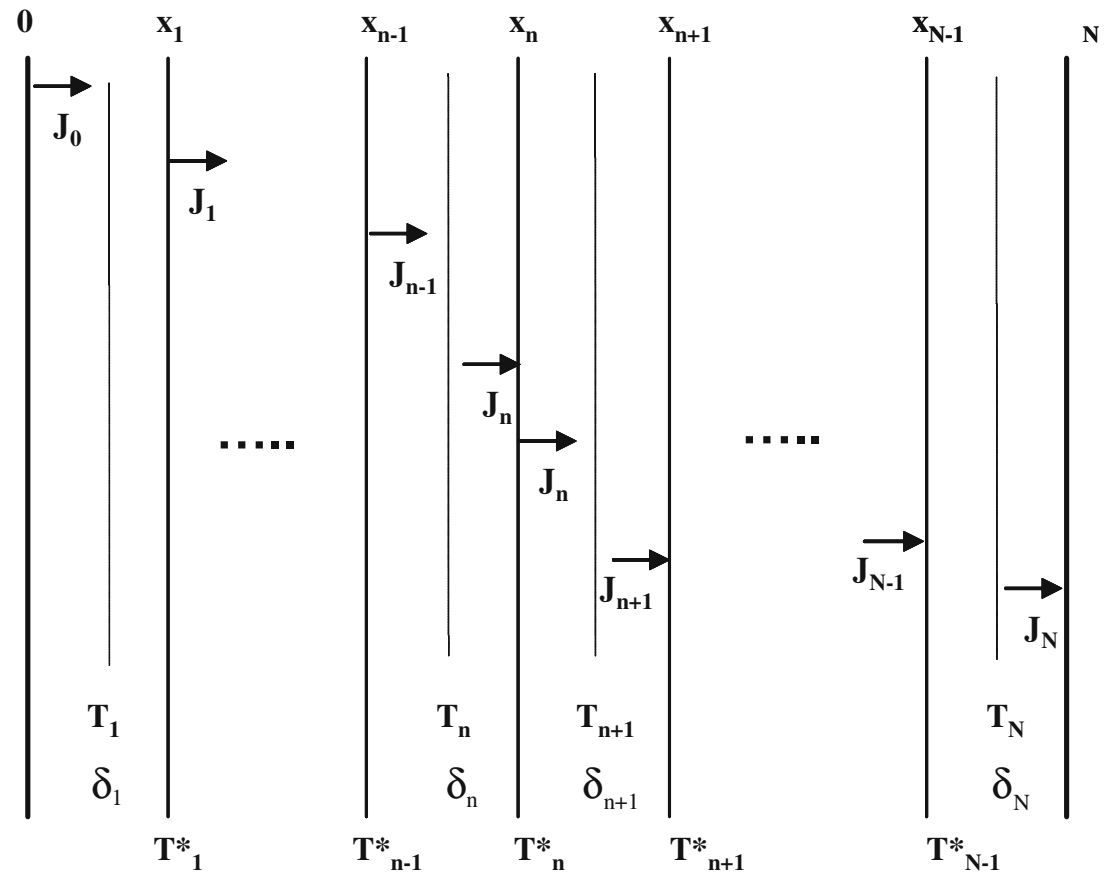

Fig. 2. Schematic diagram of the system used for the numerical implementation and its mesh. 
At the boundary $n=N$ the temperature is kept constant, $T_{N}=T_{L}$; therefore, at the point $N-1$,

$$
\frac{\Delta T_{N-1}}{\Delta t}=D_{N-1} \eta_{N-2} T_{N-2}-D_{N-1}\left(\eta_{N-1}+\eta_{N-2}\right) T_{N-1}+q_{N-1}
$$

with

$$
q_{N-1}=D_{N-1} \eta_{N-1} T_{L}+\frac{A_{N-1}}{\left(d c_{p}\right)_{N-1}} F_{N-1}^{h} .
$$

Equations (21)-(23) are assembled into a system expressed by

$$
\frac{\Delta \vec{T}}{\Delta t}=H \vec{T}+\vec{q}
$$

where $\vec{T}(x)=\left(T_{1}, T_{2}, \ldots, T_{N-1}\right), \vec{q}(x)=\left(q_{1}, q_{2}, \ldots, q_{N-1}\right)$, and $H$ is a tridiagonal matrix of the form,

$$
\left.\begin{array}{rcccc}
H= & \multicolumn{5}{c}{} \\
\left(D_{1} \eta_{1}\right. & D_{1} \eta_{1} & 0 & 0 & 0 \\
-D_{2} \eta_{1}-D_{2}\left(\eta_{1}+\eta_{2}\right) & D_{2} \eta_{2} & 0 & 0 \\
0 & D_{3} \eta_{2} & -D_{3}\left(\eta_{2}+\eta_{3}\right) & D_{3} \eta_{3} & 0 \\
0 & 0 & D_{n} \eta_{n-1} & -D_{n}\left(\eta_{n}+\eta_{n-1}\right) & D_{n} \eta_{n} \\
0 & 0 & 0 & D_{N-1} \eta_{N-2} & -D_{N-1}\left(\eta_{N-1}+\eta_{N-2}\right)
\end{array}\right) .
$$

The transient temperature distribution in the material was calculated performing an iterative time integration using the formula,

$$
\left(1-\Delta t_{m} H_{m}\right) \vec{T}_{m+1}=\vec{T}_{m}+\Delta t_{m} \vec{q}_{m},
$$

where the index $m$ represents the time. This implicit differencing scheme is used for its superior numerical stability [9]. Strictly speaking, it would require the terms $\Delta t_{m} H_{m+1}$ and $\Delta t_{m} \vec{q}_{m+1}$, but replacing them by $\Delta t_{m} H_{m}$ and $\Delta t_{m} \vec{q}_{m}$ causes only deviations proportional to $\left(\Delta t_{m}\right)^{2}$, negligible for a sufficiently small time step.

The radiative and conductive fluxes at any time are calculated by Eqs. (7a) and (2), respectively.

\section{SIMULATION: RESULTS AND DISCUSSION}

Zirconia was taken as an example of a typical porous material used for shielding at very high temperatures. A layer of $1 \mathrm{~mm}$ thickness subjected to a laser pulse of $5 \times 10^{6} \mathrm{~J} \cdot \mathrm{m}^{-2}$ delivered in $5 \times 10^{-2} \mathrm{~s}$ (Fig. 3) was studied during the heating and cooling stages. The thermophysical properties used in the calculations are listed in Table I. The material was 


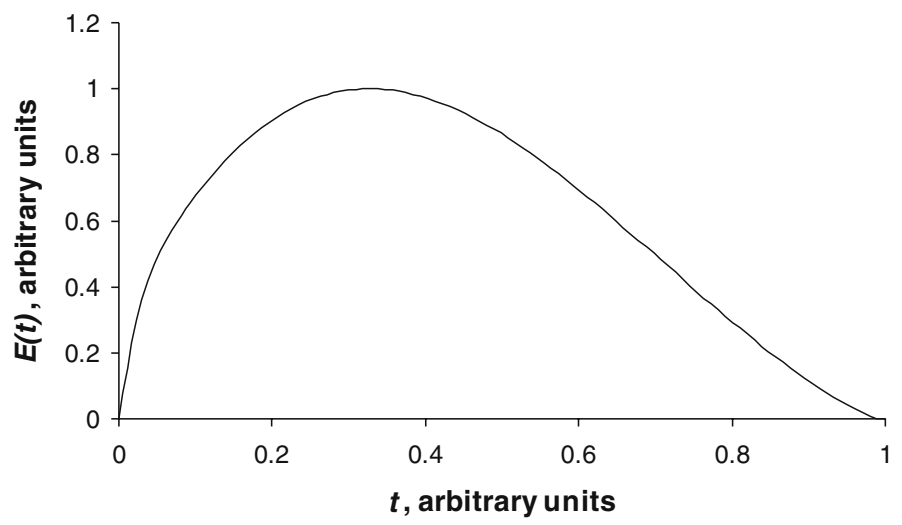

Fig. 3. Power profile of the laser pulse.

Table I. Thermophysical Properties of Zirconia

\begin{tabular}{ccc}
\hline$T(\mathrm{~K})$ & $c_{p}\left(\mathrm{~J}^{\mathrm{kg}} \mathrm{g}^{-1} \cdot \mathrm{K}^{-1}\right)[10]$ & $k\left(\mathrm{~W} \cdot \mathrm{m}^{-1} \cdot \mathrm{K}^{-1}\right)[11]$ \\
\hline 300 & 456 & 1.4 \\
400 & 517 & 1.4 \\
500 & 549 & 1.4 \\
600 & 569 & 1.5 \\
700 & 584 & 1.5 \\
800 & 596 & 1.5 \\
900 & 605 & 1.5 \\
1000 & 614 & 1.5 \\
\hline
\end{tabular}

assumed to have a density of $5.6 \times 10^{3} \mathrm{~kg} \cdot \mathrm{m}^{-3}$, an absorption coefficient $A=3.0 \times 10^{2} \mathrm{~m}^{-1}$, and a scattering coefficient $S=1.2 \times 10^{4} \mathrm{~m}^{-1}$ corresponding to a reflectivity $\rho=0.8$ of the infinitely thick layer. The optical thickness of the investigated layer was thus $W L=2.7$. A semitransparent layer was chosen for the discussion here in order to make the relationship of the different radiant fluxes visible on the same diagrams for the whole layer. The model and the algorithm, however, work equally well for optically thick layers.

The thermostat keeping the rear surface at a constant temperature $T_{L}=300 \mathrm{~K}$ was assumed to have a reflectivity $\rho_{s h}=0.2$. The initial temperature of the layer was assumed to be $300 \mathrm{~K}$. The calculations were performed with an equally spaced grid of $\delta_{n}=5.0 \times 10^{-7} \mathrm{~m}$. The total transient time of $5 \mathrm{~s}$ was investigated, the time grid consisting of more than 20,000 points and their spacing being denser for the heating stage than the 
cooling phase. In all the following figures, only representative time points are shown.

Results for the temperature profile in the layer as a function of time are shown in Fig. 4. During heating (Fig. 4) the temperature of the layer progressively increases, reaching its maximum when all the laser power is delivered. During cooling (Fig. 4b) the value of the surface temperature decreases monotonically. In the interior the temperature still increases for some time, because of the heat redistribution in the layer.

In Fig. 5 the temperature profile of the front surface is shown as a function of time. After the laser is switched off $(t=0.05 \mathrm{~s})$, the material slowly cools down, and after $4 \mathrm{~s}$, the front temperature reaches a constant value of $300 \mathrm{~K}$.

The calculated conductive heat flux is presented in Fig. 6. As already discussed above, its value is equal to zero at the front surface and rises inside the material. During heating (Fig. 6a) it reaches its maximum value at about $1 / 5$ of the layer thickness, then decreases, until close to the rear surface where it slightly increases again. During the cooling phase (Fig. $6 \mathrm{~b}$ ), the shape of the curves changes with time. First, the local minimum close to the rear surface levels out, then slowly the maximum in the first part of the curve also disappears, while simultaneously the heat flow at the rear surface increases. At approximately $1.6 \mathrm{~s}$ the curve shows a monotonically decreasing behavior with position. After this time the heat flow keeps its monotonically decreasing shape.

In Fig. 7 the quantity $F^{\mathrm{h}}$, proportional to the volumetric heat source, is reported. It monotonically increases and decreases during heating and cooling, respectively.

The calculated radiative fluxes are shown in Fig. 8. During heating (Fig. 8a) $J_{\text {rad }}$ reaches its maximum value at the front surface and decreases monotonically with position. During cooling (Fig. 8b) the layer is divided into two zones, a zone where the radiative flow is negative (the flow is directed towards the front surface) and a zone where it is positive. The points where $J_{\text {rad }}=0$ move with time to the rear surface. After about $2 \mathrm{~s}$, the total flow is negative.

In Fig. 9 the radiative and conductive fluxes are compared at two representative times. It can be seen that during heating (Fig. 9a) radiation is the dominant mode of energy transfer while during cooling (Fig. 9b) the dominant mode is conduction. The negative values of $J_{\text {rad }} / J_{Q}$ in the first half of the layer are due to the fact that in this zone the radiative flux is directed towards the front surface. 

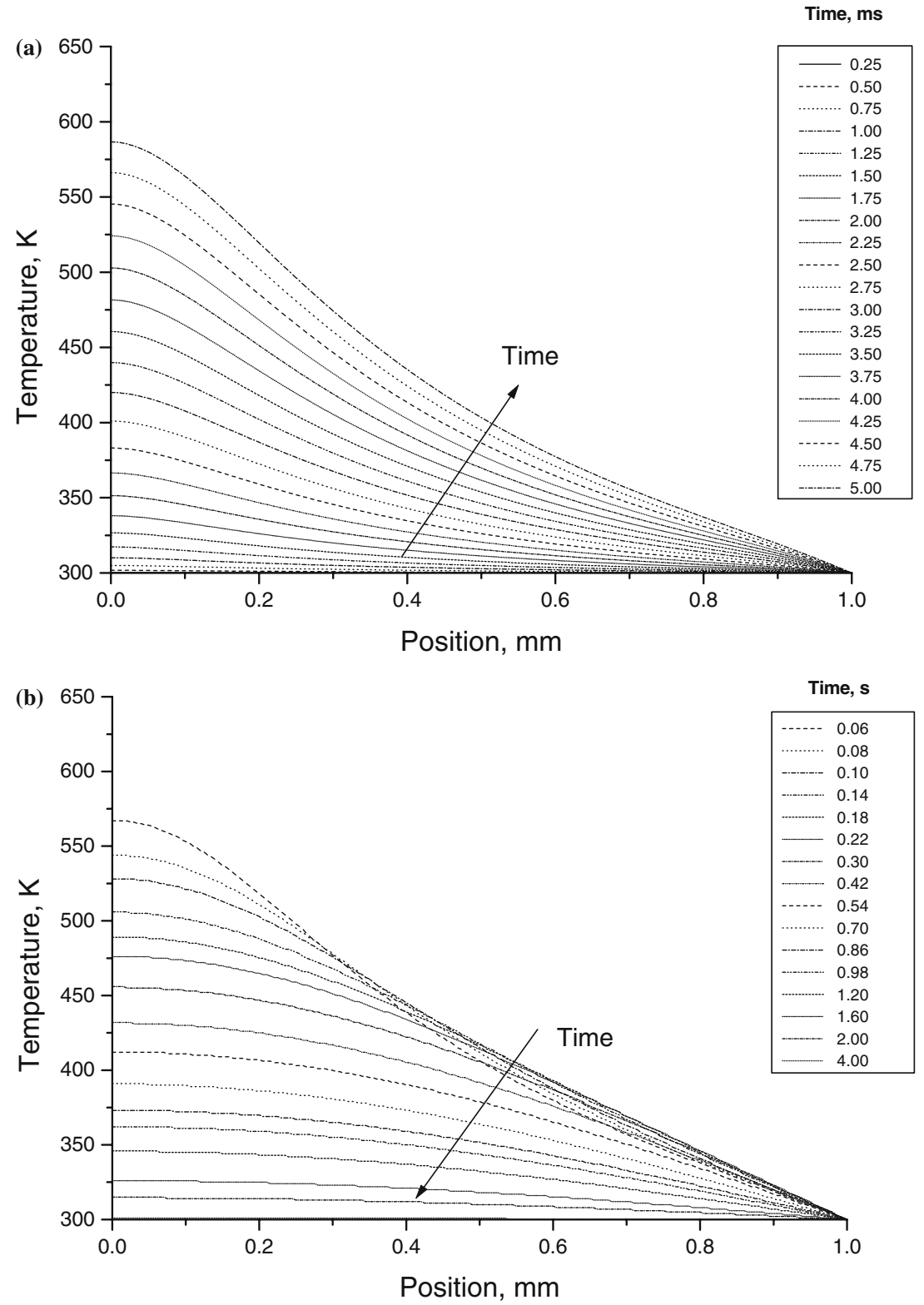

Fig. 4. Transient temperature distribution: (a) heating, during the laser pulse and (b) cooling, after the laser has been switched off. 


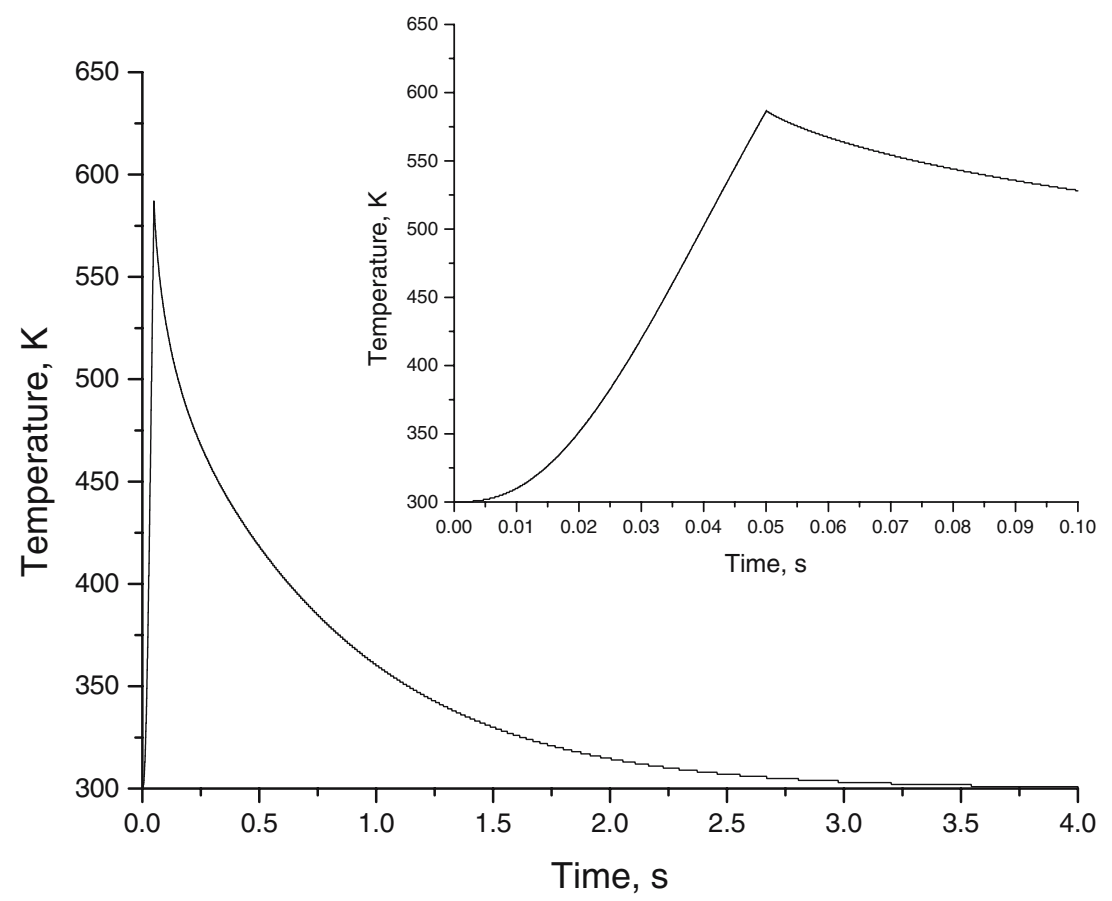

Fig. 5. Transient temperature of the front surface. Temperature reaches its maximum value at $50 \mathrm{~ms}$, when the laser pulse ends.

\section{CONCLUSIONS}

A new approach for solving the transient equation of energy transfer for a conductive and radiative medium has been presented. The problem of an infinitely extended, conductive and radiative layer subjected to a laser pulse on one face and kept at constant temperature on the other one has been treated. The radiative field is described with the two-flux approximation, and the solution of the radiative transfer equation is expressed as the sum of a special solution of the full, inhomogeneous equation plus a solution of the homogeneous equation (the equation without the source term). The special solution, for which an explicit expression is derived in terms of the temperature field, is dominant in the interior of the participating medium and takes into account the source term, while with the second contribution, the boundary conditions for the spectral radiance can be matched.

With this method, a considerable reduction of the expense for the numerical calculation is achieved. In fact, it essentially amounts to numer- 
(a) Time, ms

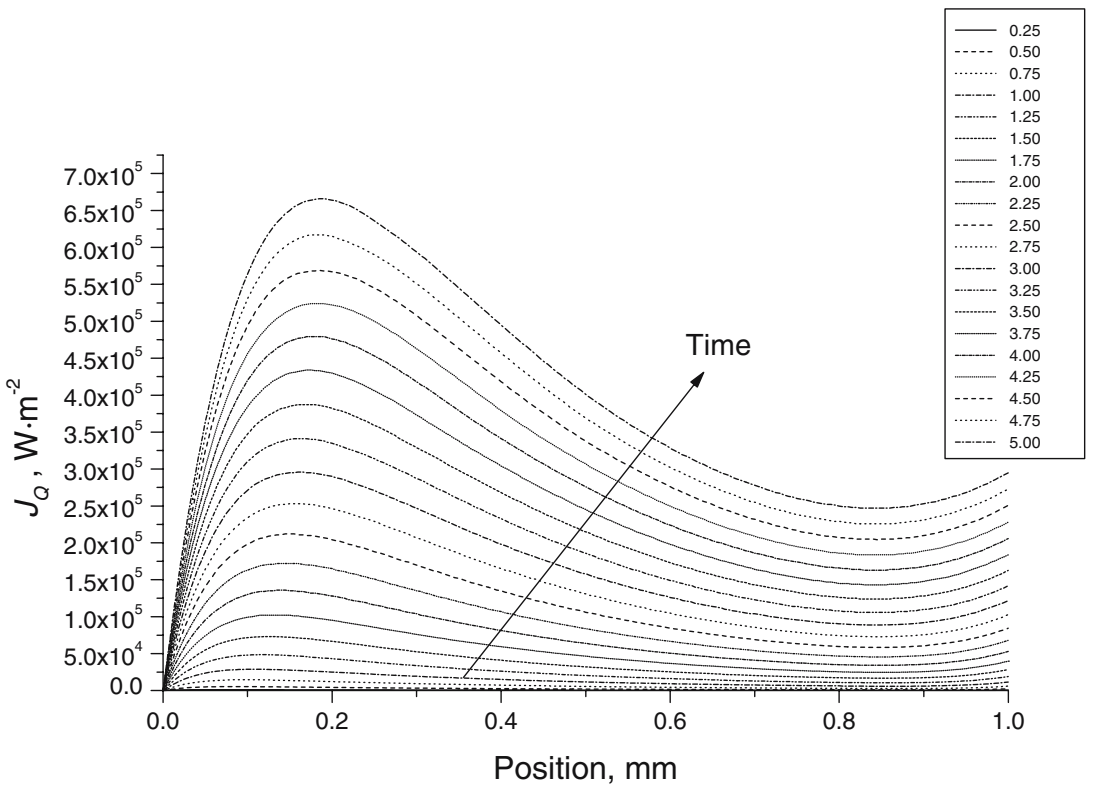

(b)

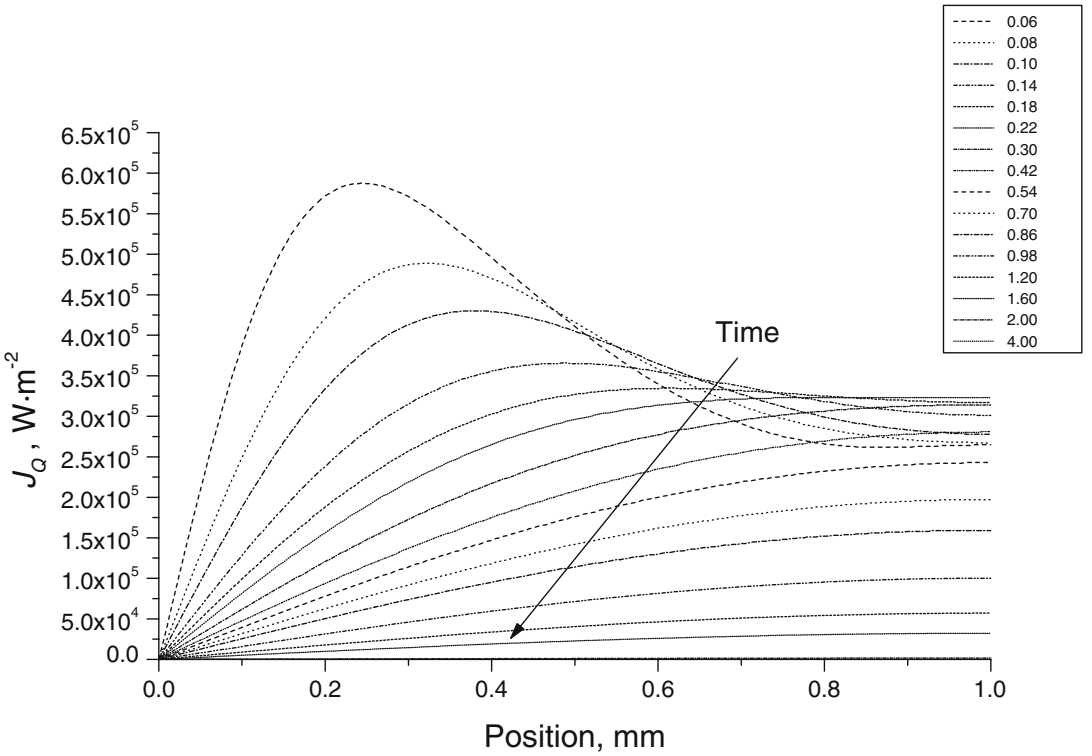

Fig. 6. Conductive flux in the layer: (a) heating, during the laser pulse and (b) cooling, after the laser has been switched off. 

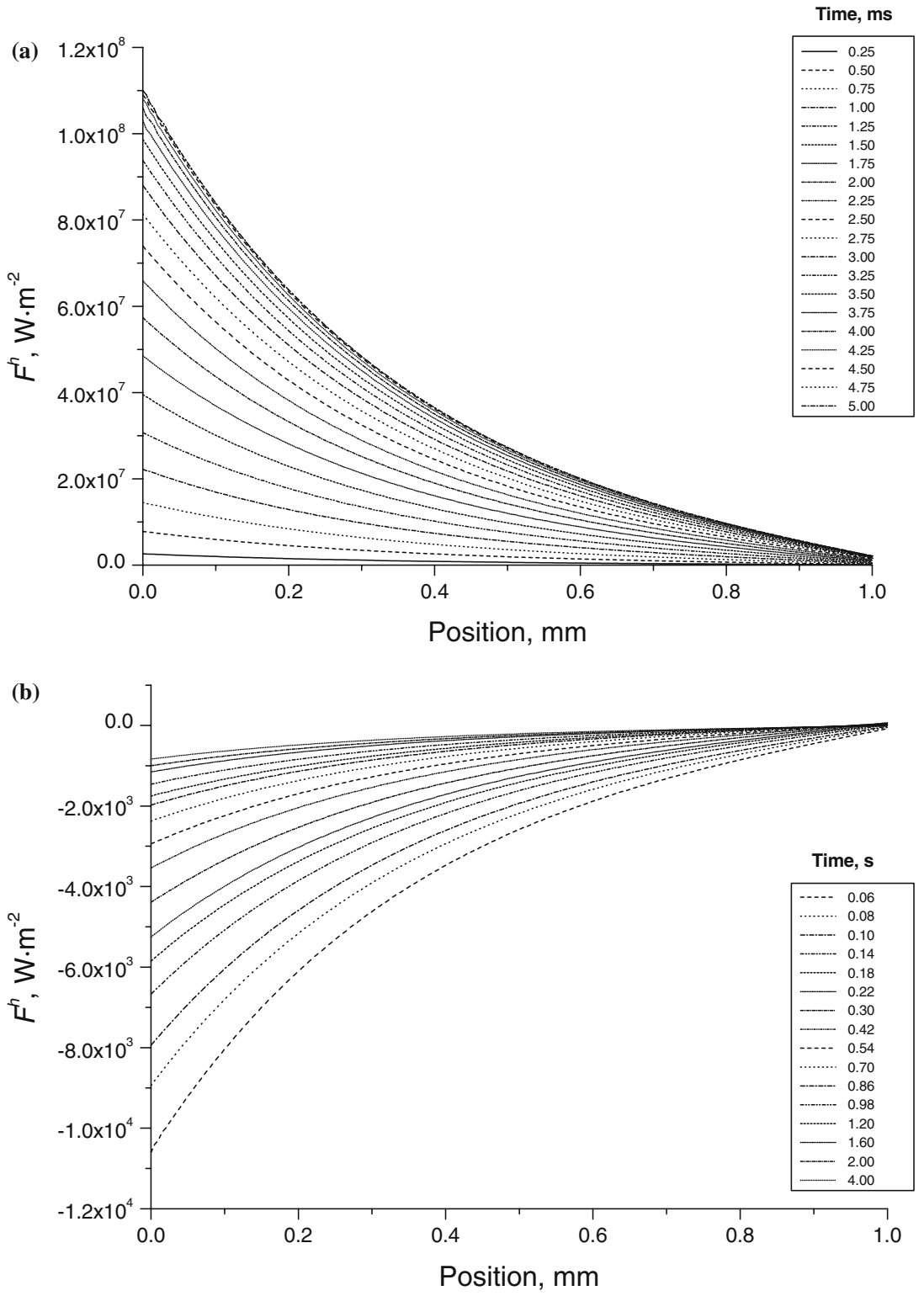

Fig. 7. $F^{\text {h }}$, quantity proportional to the source strength in the differential equation, Eq. (15): (a) heating, during the laser pulse and (b) cooling, after the laser has been switched off. 


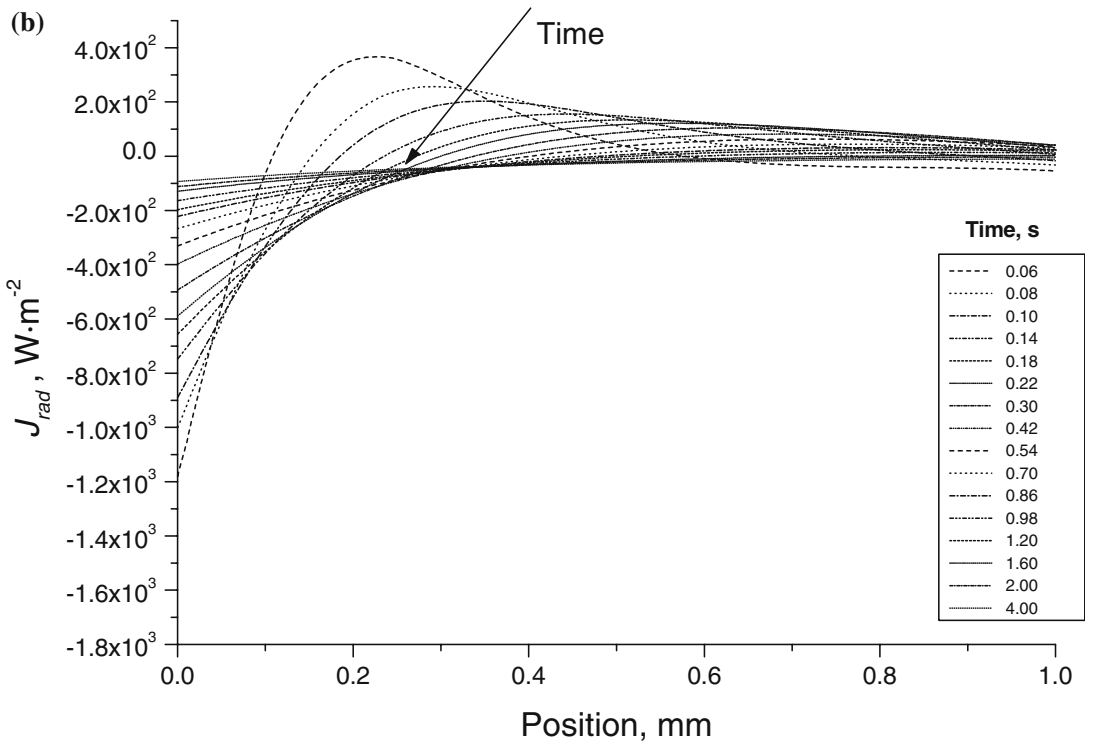

Fig. 8. Net radiative flux in the layer: (a) heating, during the laser pulse and (b) cooling, after the laser has been switched off. 


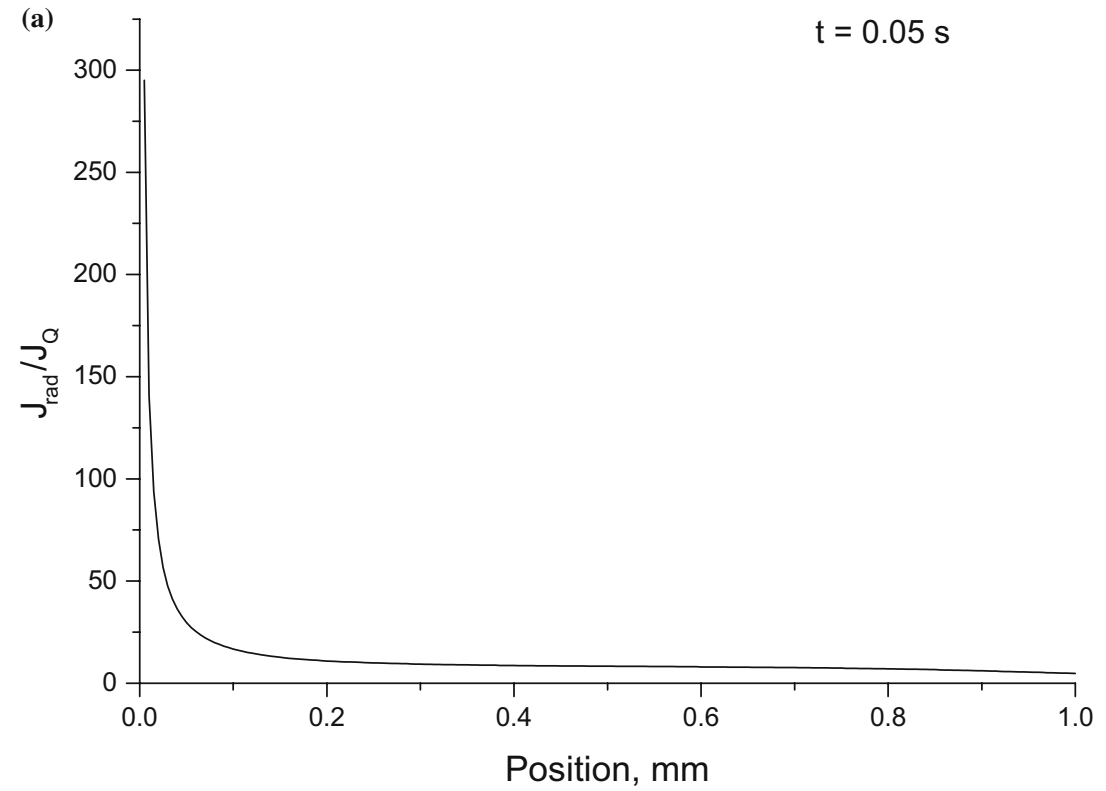

(b)

$$
t=0.5 \mathrm{~s}
$$

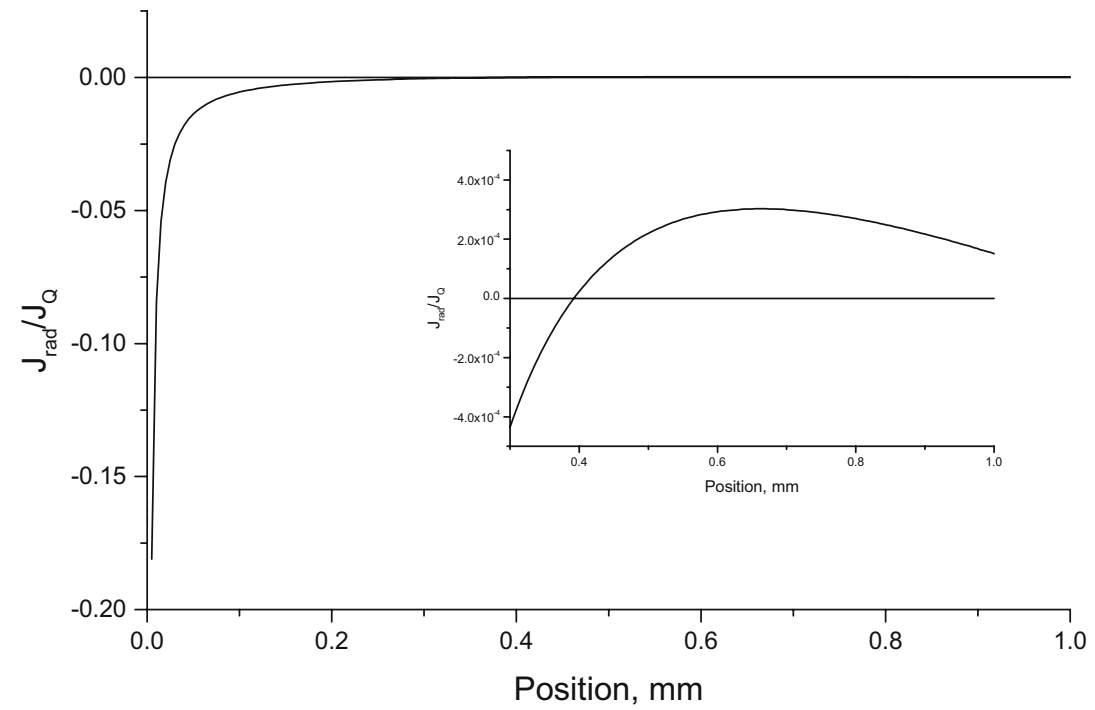

Fig. 9. Comparison of radiative and conductive fluxes in the layer at two representative times: (a) during heating, $t=0.05 \mathrm{~s}$ and (b) during cooling, $t=0.5 \mathrm{~s}$. 
ically solving a heat conduction problem with a temperature-dependent thermal conductivity and a volumetric source term.

The newly developed model has been implemented in a computer code, and the transient energy transfer for a thin layer of zirconia subjected to laser heating has been simulated.

\section{ACKNOWLEDGMENT}

This work has been supported by the Swiss Federal Office of Energy (BFE).

\section{NOMENCLATURE}

$A$ absorption coefficient, $\mathrm{m}^{-1}$

$c_{p} \quad$ specific heat, $\mathrm{J} \cdot \mathrm{kg}^{-1} \cdot \mathrm{K}^{-1}$

$d$ density, $\mathrm{kg} \cdot \mathrm{m}^{-3}$

$E(t) \quad$ laser energy, $\mathrm{J} \cdot \mathrm{m}^{-2}$

$F \quad J_{+}+J_{-}$, auxiliary flux quantity, $\mathrm{W} \cdot \mathrm{m}^{-2}$

$F^{\mathrm{h}}$ auxiliary flux quantity, solution of the homogeneous equation, $\mathrm{W} \cdot \mathrm{m}^{-2}$

$F^{\text {si }}$ auxiliary flux quantity, solution of the inhomogeneous equation, $\mathrm{W} \cdot \mathrm{m}^{-2}$

$J_{+}, J_{-}$radiative fluxes in the + and - directions, $\mathrm{W} \cdot \mathrm{m}^{-2}$

$J^{\text {h }} \quad$ flux quantity, solution of the homogeneous equation, $\mathrm{W} \cdot \mathrm{m}^{-2}$

$J^{\mathrm{si}} \quad$ flux quantity, solution of the inhomogeneous equation, $\mathrm{W} \cdot \mathrm{m}^{-2}$

$J_{Q} \quad$ conductive flux, $\mathrm{W} \cdot \mathrm{m}^{-2}$

$J_{\text {rad }} \quad$ radiative flux, $\mathrm{W} \cdot \mathrm{m}^{-2}$

$k$ thermal conductivity, $\mathrm{W} \cdot \mathrm{m}^{-1} \cdot \mathrm{K}^{-1}$

$L \quad$ thickness of the plane layer, $\mathrm{m}$

$S \quad$ scattering coefficient, $\mathrm{m}^{-1}$

$t \quad$ time, s

$T \quad$ absolute temperature, $\mathrm{K}$

$\Delta t_{m} \quad t_{m}-t_{m-1}$, step for time integration, $\mathrm{s}$

$\delta_{n} \quad x_{n}-x_{n-1}$, space grid step at point $n, 0 \leqslant n \leqslant N, \mathrm{~m}$

$\rho \quad$ reflectivity of the medium

$\rho_{s h} \quad$ reflectivity of the thermostat

$\sigma_{\mathrm{B}} \quad$ Stefan-Boltzmann constant, $\mathrm{W} \cdot \mathrm{m}^{-2} \cdot \mathrm{K}^{-4}$ 


\section{REFERENCES}

1. R. Siegel and J. R. Howell, Thermal Radiation Heat Transfer, $4^{\text {th }}$ Ed. (Taylor and Francis, New York, 2002), pp. 625-662.

2. R. Siegel, J. Heat Transfer 120:4 (1998).

3. R. Siegel, Int. J. Eng. Sci. 36:1701 (1998).

4. O. Hahn, F. Raether, M. C. Arduini-Schuster, and J. Fricke, Int. J. Heat Mass Transfer 40:689 (1997).

5. L. K. Matthews, R. Viskanta, and F. P. Incropera, J. Solar Energy Eng. 107:29 (1985).

6. H. Tan, L. Ruan, X. Xia, Q. Yu, and T. W. Tong, Int. J. Heat Mass Transfer 42:2967 (1999).

7. R. G. Siddall, Proc. $4^{\text {th }}$ Symp. Flames and Industry (Imperial College, London, The Institute of Fuel, 1972), pp. 169-177.

8. W. W. Wendlandt and H. G. Hecht, Reflectance Spectroscopy (John Wiley, New York, 1966), pp. 55-65.

9. W. H. Press, S. A. Teukolsky, W. T. Vetterling, and B. P. Flannery, Numerical Recipes in $C$, 2nd Ed. (Cambridge University Press, 1996), pp. 734-747.

10. J. P. Coughlin and E. G. King, J. Am. Chem. Soc. 72:2262 (1950).

11. V. Y. Checkhovskoy and A. M. Banaev, in Heat and Mass Transfer, Vol. VII, A.V. Likov, ed. ("Nauka I. Tekhnika," URSS, Minsk, 1968), p. 591. 